\title{
Some insights into the structural relaxation of spin-cast, glassy polymer thin films
}

\author{
Izaro López García ${ }^{1}$, Joseph L Keddie ${ }^{1}$ and Michele Sferrazza ${ }^{2}$
}

Polymer Journal (2011) 43, 214-217; doi:10.1038/pj.2010.123; published online 8 December 2010

Keywords: non-equilibrium; polymer thin films; relaxation; solvent loss; spin-casting; thermal expansion

The widespread use of thin films in a range of applications and industries, from coatings, inks and lithography to nano-imprinting, optoelectronics and memory devices, ${ }^{1}$ has made the understanding of thin films, particularly the changes induced by structural relaxation and solvent evaporation, very important. There is a need to know whether a film will change in dimensions after its deposition and how fast these changes will occur. Physical aging of thin polymer systems is, more generally, an important phenomenon in polymer physics: when a polymer is cooled quickly below its glass transition temperature $\left(T_{\mathrm{g}}\right)$, at which point freezing of the matrix takes place, ${ }^{2}$ it is in a nonequilibrium state. When it is aged at temperatures below $T_{\mathrm{g}}$, a structural relaxation toward the equilibrium volume can take place. $^{2,3}$ (The equilibrium volume represents the volume obtained through infinitely slow cooling.) Recent works have shown that the structure, wetting and mobility of polymers in thin films can differ greatly from those of the bulk. ${ }^{3-5}$ For example, a well-documented property of thin polymer films that exhibits thickness dependence is its $T_{\mathrm{g} .}{ }^{6,7}$

In many applications, thin films are prepared by depositing a dilute solution of polymer onto a substrate; thereafter, the substrate is spun at high velocity, at which the solution spreads, thereby ejecting any excess off the substrate. ${ }^{8}$ Subsequently during this process, as the solvent evaporates, the polymer volume fraction, $\phi_{\text {pol }}$, increases while at the same time the $T_{\mathrm{g}}$ of the solution also increases and approaches the experimental temperature. When the solution's $T_{\mathrm{g}}$ reaches the experimental temperature, the solution forms a glass. The film is then in a state of non-equilibrium, the structure having been 'frozen in., 3,9 This current work is with regard to the relaxation that occurs in polymers that have undergone such a 'solvent quench,' rather than a thermal quench, through glass transition.

Two poly(butyl methacrylate) polymers have been used in our study: $\mathrm{P}$ (normal-BMA) and $\mathrm{P}$ (tertiary-BMA). These isomers have a large difference in their $T_{\mathrm{g}}$ values, whereas elemental compositions of their repeat unit are identical and therefore differences in interactions with solvent or silicon substrate are not expected to affect the experiments. $\mathrm{P}$ (norm-BMA) has a $T_{\mathrm{g}}$ of $20^{\circ} \mathrm{C}$, ${ }^{10}$ which is below the experimental temperature of $\sim 23^{\circ} \mathrm{C}$; therefore, it remains rubbery throughout the experiments. In contrast, $\mathrm{P}\left(\right.$ tert-BMA) has a $T_{\mathrm{g}}$ of $107^{\circ} \mathrm{C}$, below which the polymer forms a glass and is subject to relaxation. Hence, $\mathrm{P}$ (tert-BMA) and $\mathrm{P}$ (norm-BMA) films were used to explore the differences between rubbery and glassy behavior.

The polymers were provided by Polymer Source Inc. (Quebec, CA, USA) and used as received. The number-average molecular weights $\left(M_{\mathrm{n}}\right)$ of the two polymers were comparable, being $182 \mathrm{~kg} \mathrm{~mol}^{-1}$ for $\mathrm{P}$ (norm-BMA) (polydispersity $\left(M_{\mathrm{w}} / M_{\mathrm{n}}\right)$ of 1.02$)$ and $138 \mathrm{~kg} \mathrm{~mol}^{-1}$ for $\mathrm{P}$ (tert-BMA), with $M_{\mathrm{w}} / M_{\mathrm{n}}=1.06$. In all experiments, thin films were deposited by spin-casting solutions in toluene at a rate of 2000 r.p.m. for $30 \mathrm{~s}$. The substrates were single-crystal (100) silicon wafers, used as received such that the native oxide was on the surface. Film thickness was kept constant at $130 \mathrm{~nm}$ to minimize thickness effects. Films were aged at room temperature $\left(23^{\circ} \mathrm{C}\right)$ under vacuum for different periods up to $24 \mathrm{~h}$, to allow the solvent to leave the film and for the structures to relax.

Ellipsometry measurements (VASE, J.A. Woollam Co., Lincoln, NE, USA) were taken as a function of time over multiple wavelengths, selected to provide the greatest sensitivity. The film samples were placed on the ellipsometer stage previously fitted with a heating stage (Linkam, Surrey, UK). During the measurements, films were heated in air at a constant temperature increase of $2{ }^{\circ} \mathrm{C} \mathrm{min}-1$ from room temperature $\left(\sim 23^{\circ} \mathrm{C}\right)$ up to a maximum of $150^{\circ} \mathrm{C}$ (chosen to avoid any thermal decomposition of the polymer ${ }^{11}$ ). The films were then cooled at an equal but opposite rate of $-2{ }^{\circ} \mathrm{C} \mathrm{min}^{-1}$. The ellipsometric angles $\Psi$ and $\Delta$ were measured as a function of time. Fitting of data using a Marquardt algorithm was performed to obtain the thickness, $h$, and refractive index, $n$, of the film as a function of time. Time was correlated to temperature through the heating rate.

${ }^{1}$ Department of Physics, University of Surrey, Surrey, UK and 2Département de Physique, Université Libre de Bruxelles, Bruxelles, Belgium 
The fitting parameters were thickness, $h$, and parameter $A$ of the refractive index in the Cauchy dispersion model, ${ }^{8,12} n(\lambda)=A+\left(B / \lambda^{2}\right)$, where $B$ is a material constant and $\lambda$ is the wavelength of the incident light.

Relaxation processes are normally observed in bulk polymers by measuring the volume of the glass, $V_{\mathrm{t}}$, at a given time. The relative departure from the equilibrium volume, $\delta_{\mathrm{V}}$, is then defined as $\delta_{\mathrm{V}}=\left(V_{\mathrm{t}}-V_{\infty}\right) / V_{\infty}$, where $V_{\infty}$ represents the equilibrium volume obtained after long relaxation times. ${ }^{2}$ In an analogy to bulk behavior, the relaxation in thin films can be monitored by measuring the thickness of the glass, $h$, at a given time (and temperature). A final equilibrium value for thickness, $h_{\infty}$, is obtained from a film after heating and cooling to room temperature. Its value is obtained from ellipsometry in a full spectroscopic scan from 400 to $800 \mathrm{~nm}$. It was found that the thickness changes occurring over time after a heatingcooling cycle were negligible in comparison with that found during the cycle. The relative departure from equilibrium, defined as the excess thickness normalized with respect to $h_{\infty}$ at room temperature, was calculated as $\delta_{\mathrm{RT}}=\left(h-h_{\infty}\right) / h_{\infty}$. In these experiments in which temperature is not constant, $\delta_{\mathrm{RT}}$ also contains information on the thickness increase arising from thermal expansion. Using a measurement of the initial film thickness after spin-casting, $h_{0}$, the initial departure from equilibrium thickness, $\delta_{0}$, can be defined as $\delta_{0}=\left(h_{0}-h_{\infty}\right) / h_{\infty}$.

Figure 1 shows the variation of $\delta_{\mathrm{RT}}$ as a function of temperature for the $\mathrm{P}$ (norm-BMA) isomer (top figure), which is a rubbery polymer at

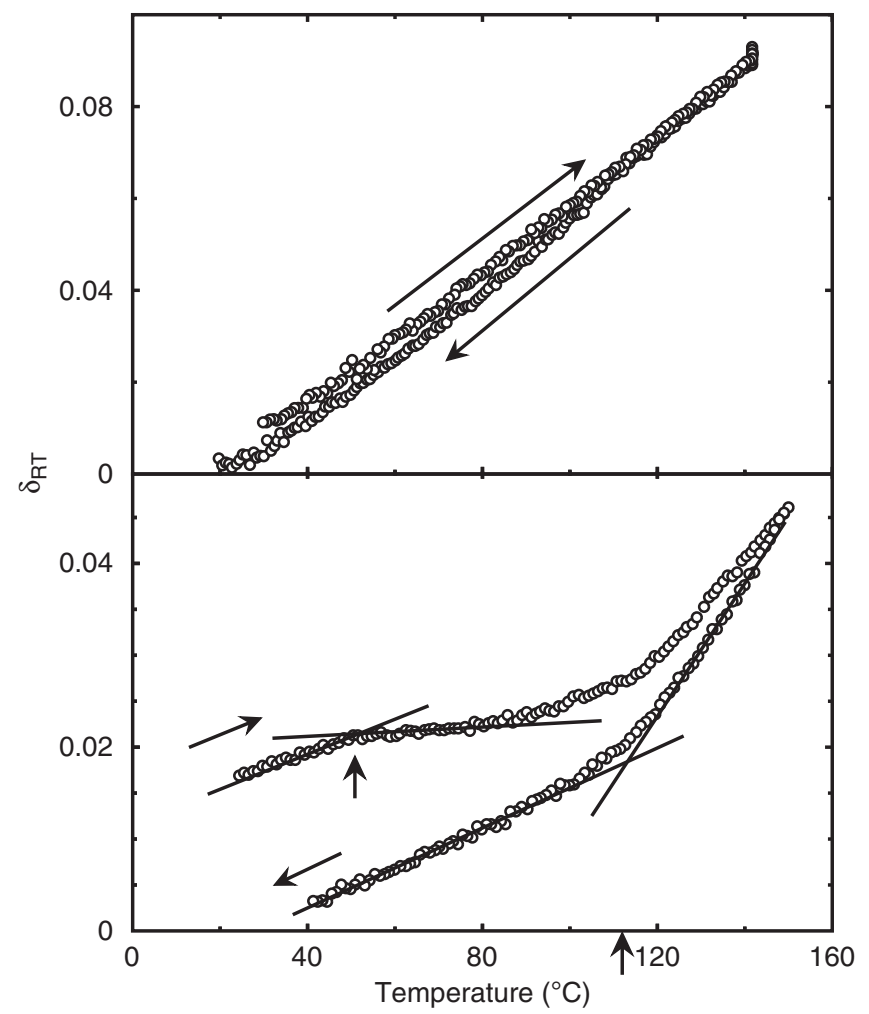

Figure 1 The relative departure from room temperature equilibrium, $\delta_{\mathrm{RT}}$, as a function of temperature. Top: $P($ norm-BMA) film heated from room temperature to $130{ }^{\circ} \mathrm{C}$ at $2{ }^{\circ} \mathrm{Cmin}^{-1}$, then cooled to room temperature at the same rate. No glass transition is seen at any temperature, and heating and cooling curves overlap almost perfectly. Bottom: the same type of data obtained from a glassy $\mathrm{P}$ (tert-BMA) film shows a departure from linearity and a large difference between the heating and cooling curves. The apparent $T_{\mathrm{g}}$ on cooling is indicated by arrows. room temperature. The linear thermal expansion coefficient $\alpha=1$ / $h(\partial h / \partial T)_{P, t}$ was calculated from a linear fit to measurements of thickness as a function of temperature.

A value of $\alpha=7.3 \times 10^{-4} \mathrm{~K}^{-1}$ was obtained, which agrees with the literature value $\mathrm{e}^{10}$ of $7.0 \times 10^{-4} \mathrm{~K}^{-1}$. (Note that if thermal expansion is restricted in the plane of the film, one would expect to see a higher expansivity in the direction normal to the plane. ${ }^{13}$ Thus, in this materials system, there is no evidence that an attraction between the polymer and substrate is preventing slippage of the polymer along the substrate.) It was found that the thickness dependence on temperature was essentially identical during the heating and cooling stages. There is no evidence for structural relaxation, as is expected for a rubbery polymer. The superposition of the heating and cooling curves supports the conclusion that the rubbery polymer retains no solvent, which would lead to a volume change upon its liberation. This result clearly agrees with the ideas proposed by Leibler and Sekimoto ${ }^{11}$ that an important factor in solvent retention in polymers must be the increase in bulk modulus of the polymer as it undergoes transition from the rubbery to the glassy phase. They proposed that the chemical potential of the solvent in a polymer solution should be modified from the standard Flory-Huggins expression when the polymer becomes a glass, adding an additional term, which is a function of the polymer bulk modulus.

On the other hand, Figure 1 (bottom part) shows temperaturedependent data obtained from $\mathrm{P}\left(\right.$ tert-BMA), having a $T_{\mathrm{g}}$ of $107^{\circ} \mathrm{C}$, that is well above room temperature. Initially, as the temperature rises, the film exhibits a thermal expansion that is broadly consistent with the literature values for a polymer glass expansion coefficient.

If the film contained no solvent, we would expect the rising trend to continue until it approached the $T_{\mathrm{g}}$ of the neat polymer glass, at which point the expansivity would increase toward that of the rubbery state. However, this is clearly not the case, as the film's expansion is arrested when reaching $47 \pm 3{ }^{\circ} \mathrm{C}$, which is interpreted as being an apparent glass transition temperature (arrow in the figure) of the glassy polymer matrix. If we consider that there is some solvent trapped in the polymer matrix, then, below this temperature, the solvent is unable to leave the film as this would require a compression of the matrix (assuming no voids are allowed to be created because of the associated rise in surface energy). According to the Kelley-Bueche polymer/ solvent description, ${ }^{14}$ the presence of solvent (with a low $T_{\mathrm{g}}$ ) in a polymer depresses its $T_{\mathrm{g}}$ as a function of the amount of solvent present. ${ }^{14,15}$ When this lower glass transition temperature is reached, the distortion energy ${ }^{2,15}$ is overcome and trapped solvent is released, allowing the film to decrease in thickness while at the same time raising the polymer/solvent $T_{\mathrm{g}}$ further. During this period, both phenomena compete: thermal expansion increases the thickness, whereas solvent loss decreases it. On reheating the film in a second cycle (data not shown), the thermal expansivity follows the same paths for the glassy and rubbery states that were observed in the first cooling curve. This result indicates that relaxation and the accompanying solvent loss are complete after the initial heating.

These differences in relaxation and expansion behavior observed between glassy and rubbery polymers have not been demonstrated explicitly in thin films in any previous work that we have seen.

Figure 1 clearly shows this period as expansion is arrested until temperatures approach the bulk $T_{\mathrm{g}}$ of the polymer. Cooling data shown in Figure 1 can accurately be used to calculate values such as the $T_{\mathrm{g}}$ of the neat polymer $\left(112 \pm 3^{\circ} \mathrm{C}\right.$ calculated, $107^{\circ} \mathrm{C}$ literature value ${ }^{10}$ ), and thermal expansion coefficient of the glassy phase $\left(\alpha_{\mathrm{g}}=2.0 \times 10^{-4} \mathrm{~K}^{-1}\right.$ calculated, $\alpha_{\mathrm{g}}=2.80 \times 10^{-4} \mathrm{~K}^{-1}$ literature value) and the rubbery phase $\left(\alpha_{\mathrm{r}}=7.4 \times 10^{-4} \mathrm{~K}^{-1}\right.$ calculated, 


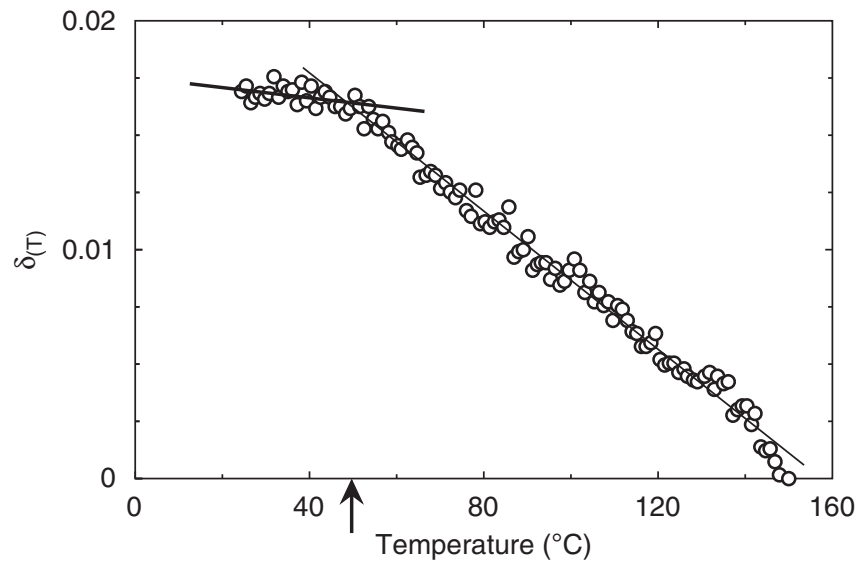

Figure 2 The temperature-dependence of the relative departure from equilibrium thickness (corrected for temperature), $\delta(T)$, for a $\mathrm{P}$ (tert-BMA) thin film. It can be clearly seen that $\delta(T)$ remains constant until $47^{\circ} \mathrm{C}$ is reached as shown by the arrow. At that temperature, the film starts to relax, seen as a decrease in $\delta(T)$. One interpretation is that it has reached its apparent glass transition temperature.

$\alpha_{\mathrm{r}}=7.20 \times 10^{-4} \mathrm{~K}^{-1}$ literature value). ${ }^{10}$ Sufficiently good agreement with the literature values contributes to our confidence in interpreting the data obtained.

Consideration of the relative departure from equilibrium, $\delta$, is best suited for isothermal experiments. To gain an insight into the temperature dependence of the fractional 'excess volume', beyond the equilibrium volume, a better approach is to calculate the departure from equilibrium thickness at a given temperature, relative to the equilibrium thickness at that same temperature, as $\delta(T)=\left[h(\mathrm{~T})-h_{\infty}(T)\right] / h_{\infty}(T)$. In this case, the $h_{\infty}$ values were taken from the cooling curve. When $\delta(T)$ is plotted as in Figure 2, it is observed that it is unchanged until a certain temperature $\left(47^{\circ} \mathrm{C}\right)$ is reached, at which point the film is able to relax. This finding is consistent with the idea that the concentration of solvent in the film lowers its $T_{\mathrm{g}}$ from that of the neat polymer. Only at the apparent glass transition temperature does $\delta(T)$ start to decrease. We can use the apparent glass transition temperature to obtain the solvent concentration required in the film to depress the temperature from the bulk value. To lower the glass transition temperature to $47 \pm 3{ }^{\circ} \mathrm{C}$, the solvent volume fraction needs to be $0.140 \pm 0.011$. (This calculation assumes a value for the $T_{\mathrm{g}}$ of toluene taken as $2 / 3$ of the crystal melting temperature and uses the Kelley-Bueche polymer/solvent equation. ${ }^{14}$ )

Follow-up experiments were carried out to examine the relaxation processes in blends of $\mathrm{P}$ (tert-BMA) and poly(methyl methacrylate) (PMMA). Three different $\mathrm{P}$ (tert-BMA)/PMMA blend ratios of 9:1, 1:1 and 1:9 were measured. Samples were aged in vacuum for different times at room temperature before analysis. The departure from room temperature equilibrium, $\delta_{\mathrm{RT}}$, was measured with ellipsometry when the system was heated at a constant rate of $2{ }^{\circ} \mathrm{C} \mathrm{min}^{-1}$ for blends with aging times ranging from 0 to $96 \mathrm{~h}$. Figure 3 shows, as an example, $\delta_{\mathrm{RT}}$ as a function of temperature for the $\mathrm{P}($ tert-BMA)/PMMA blend with a ratio of 1:9 for a range of times aged in vacuum at room temperature. When the films are heated above an apparent $T_{\mathrm{g}}$, there is a structural relaxation leading to a decrease in film thickness. Once the film has fully relaxed and reached equilibrium at the experimental temperature, the thickness starts to increase again, as is expected according to its thermal expansivity. When the film is aged for longer, the departure from equilibrium and the amount of relaxation decrease.

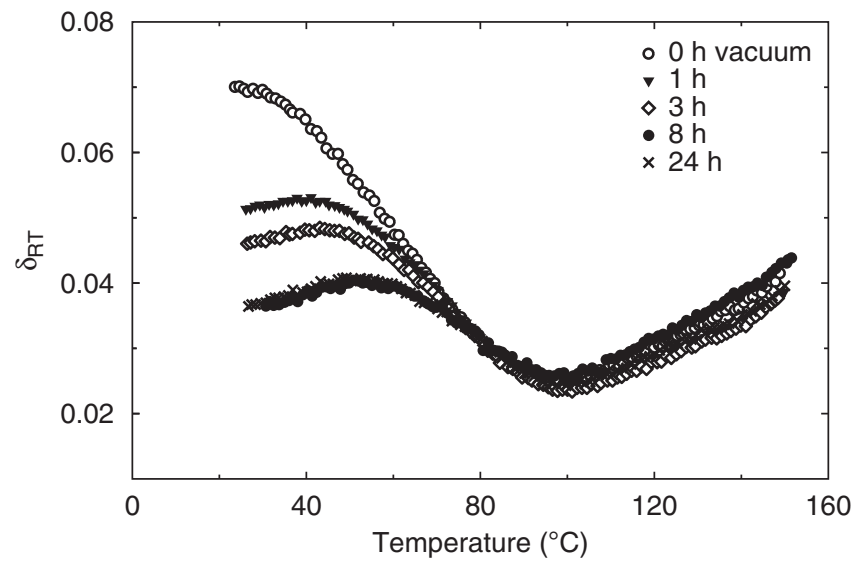

Figure 3 The relative departure from equilibrium, $\delta_{\mathrm{RT}}$, as a function of $T$. Heating curves are shown for $\mathrm{P}$ (tert-BMA)/PMMA (1:9 ratio) blend films aged in vacuum over a range of time.

Little or no change is appreciable as the aging time is increased both for pure $\mathrm{P}$ (tert-BMA) and for $\mathrm{P}$ (tert-BMA)/PMMA with a ratio of 9:1 (data not shown). The results differ strongly for PMMA-rich films with a $\mathrm{P}$ (tert-BMA)/PMMA ratio of 1:1 and 1:9. We observe in freshly cast (non-aged) films that, as the fraction of PMMA is increased in the blend, the film exhibits a higher initial departure from its equilibrium. When a film is aged at room temperature, structural relaxation occurs-albeit very slowly_before the heating of the film. Hence, the amount of relaxation observed as the film is heated decreases with increasing aging time. This concept explains why volume relaxation is greatly affected by the temperature of the system ${ }^{16}$ and the time of aging. Similar behavior has been observed in many studies in literature on bulk polymers, ${ }^{2,16,17}$ but few reports have explored these phenomena in thin film systems. ${ }^{18}$ The data seem to suggest that $\mathrm{P}$ (tert-BMA)-rich films relax very fast during the deposition procedure, such that they obtain an equilibrium thickness soon after deposition. In contrast, PMMA films undergo a significant amount of slow relaxation.

Further analysis was used to identify differences between the polymers. A volume relaxation rate, $\beta$, can be defined in terms of the slope of isothermal measurements of $\delta$ as ${ }^{16} \beta=\mathrm{d} \delta / \operatorname{dog}\left(t_{i}-t_{0}\right)$, where $t_{0}$ is initial time, $t_{i}$ is the time at point $i$ and $\delta$ is as defined previously. This relaxation rate can be compared for in the different systems to obtain some insight into the relation between the isothermal relaxation rate and the compositions of the different polymer blends. For each of the compositions, the gradient of $\delta$ (obtained isothermally at room temperature) plotted against the logarithm of time was used to obtain a value of the isothermal relaxation rate, $\beta$, at room temperature. The measurements of $\beta$ and $\delta_{0}$ for these different systems are reported in Table 1 .

On considering the trend in $\beta$, it is apparent that PMMA-rich films exhibit the largest values compared with other compositions. As the ratio of $\mathrm{P}($ tert-BMA) increases in the blend, the relaxation rate decreases considerably, reaching the point at which the film is pure $\mathrm{P}$ (tert-BMA) and at which the relaxation rate is the lowest. The initial departure from equilibrium is lowest for $\mathrm{P}($ tert-BMA) and increases with increasing PMMA fraction. The fact that $\mathrm{P}($ tert-BMA) has the lowest $\delta_{0}$ and also exhibits almost no relaxation for over a period of several days suggests that $\mathrm{P}$ (tert-BMA) films hold much less solvent after spin-casting from dilute solution than do PMMA-rich films. These differences are surprising considering the fact that PMMA and 
Table 1 Thermal expansivity and initial departure from equilibrium thickness values for homopolymers and polymer blends

\begin{tabular}{lcc}
\hline Polymer system & $\beta\left(K^{-1}\right)$ & $\delta_{0}$ \\
\hline Pure PMMA & $198 \times 10^{-4}$ & 0.0992 \\
PMMA 9:1 P(tert-BMA) & $113 \times 10^{-4}$ & 0.0700 \\
PMMA 1:1 P(tert-BMA) & $66 \times 10^{-4}$ & 0.0391 \\
PMMA 1:9 P(tert-BMA) & $7 \times 10^{-4}$ & 0.0200 \\
Pure P(tert-BMA) & $5 \times 10^{-4}$ & 0.0169 \\
\hline
\end{tabular}

Abbreviations: PMMA, poly(methyl methacrylate); tert, tertiary.

$\mathrm{P}$ (tert-BMA) have similar glass transition temperatures and bulk moduli.

This difference in relaxation characteristics between polymers may stem from differences in the selective character of the solvent and not from the mechanical properties of the polymers, which are similar. The $\chi$ parameter determines how the chemical potential varies with $\phi_{\text {pol }}{ }^{17}$ The $\chi$ parameter for the $\mathrm{P}($ tert-BMA $) /$ toluene system, for a polymer volume fraction of 0.8 , is around $0.25-0.3,{ }^{19-21}$ similar to that of the PS/toluene system, whereas for PMMA/toluene the value is higher, at around $0.45 .{ }^{10,18,19}$ One can argue that if the chemical potential is higher (as is the case for toluene/PMMA) and when there is some solvent in the vapor phase-such as during the spin-casting process-it will be thermodynamically favorable to have more solvent in the polymer solution. Our results are consistent with this expectation for the toluene/PMMA case in comparison with toluene/ $\mathrm{P}$ (tertBMA) or toluene/PS (H. Richardson, private communication). Despite its non-selective character, toluene is a better solvent for $\mathrm{P}$ (tert-BMA) than for PMMA.

In conclusion, the film's initial conformation after a solvent quench was explored for different polymers and their blends. In spin-cast rubbery $\mathrm{P}$ (norm-BMA) films, there is no evidence for structural relaxation on heating and no evidence for remnant solvent. On the other hand, spin-cast glassy $\mathrm{P}$ (tert-BMA) films undergo structural relaxation above an apparent $T_{\mathrm{g}}$. Comparisons between glassy $\mathrm{P}($ tert$\mathrm{BMA}$ ) and $\mathrm{PMMA} / \mathrm{P}$ (tert-BMA) with different blend ratios have shown a strong dependence of both the initial departure from equilibrium thickness, $\delta_{0}$, and the relaxation rate, $\beta$, on the $\mathrm{P}($ tertBMA) content of the film. These results indicate that $\mathrm{P}$ (tert-BMA)rich films could be losing solvent and forming dense films much faster than PMMA-rich films, such that they are closer to their equilibrium thickness when first deposited. On the other hand, the rate of structural relaxation in the solid $\mathrm{P}($ tert-BMA) film is significantly slower than in PMMA. This result shows that $\mathrm{P}($ tert-BMA) forms films with a high thickness stability, something that is attractive in practical applications, such as lithography and emerging nanoimprinted coatings. ${ }^{22}$

\section{ACKNOWLEDGEMENTS}

We gratefully acknowledge funding from the EPSRC (UK), FNRS (Belgium) and Sun Chemical, Orpington, Kent. We benefited from useful discussions with Drs H Richardson (Surrey) and D Illsley (Sun Chemical). We thank Dr de Silva (ULB) for his help with some figures.

1 Binnig, G., Despont, M., Drechsler, U., Häberle, W., Lutwyche, M., Vettiger, P., Mamin, H. J., Chui, B. W. \& Kenny, T. W. Ultrahigh-density atomic force microscopy data storage with erase capability. Appl. Phys. Lett. 74, 1329 (1999).

2 Kovacs, A. J. Transition vitreuse dans les polymères amorphes. Etude. phénoménologique. Fortschr. Hochpolym.-Forsch. 3, 394 (1964).

3 Frank, C. W., Rao, V. V., Despotopoulou, M. M., Pease, R. F. W., Hinsberg, W. D., Miller, R. D. \& Rabolt, J. F. Structure in thin and ultrathin spin-cast polymer films. Science 273, 912 (1996).

4 Reiter, G., Sharma, A., Khanna, R., Casoli, A. \& David, M. O. The strength of long-range forces across thin liquid films. J. Colloid Interface Sci. 214, 126 (1999).

5 Forrest, J. A., Svanberg, C., Révész, K., Rodahl, M., Torell, L. M. \& Kasemo, B. Relaxation dynamics in ultrathin polymer films. Phys. Rev. E 58, R1226 (1998).

6 Fukao, K. \& Miyamoto, Y. Glass transition and dynamics in thin polymer films: dielectric relaxation of thin film of polystyrene. Phys. Rev. E 61, 1743 (2000).

7 Keddie, J. L., Jones, R. A. L. \& Cory, R. A. Interface and surface effects on the glasstransition temperature in thin polymer-films. Faraday Discuss. 98, 219 (1994).

8 Richardson, H., López-García, I., Sferrazza, M. \& Keddie, J. L. Thickness dependence of structural relaxation in spin-cast, glassy polymer thin films. Phys. Rev. E 70, 051805 (2004).

9 Strobl, G. The Physics of Polymers (Springer Verlag, Berlin Heidelberg, Germany, 1997).

10 Polymer Handbook (eds Brandrup, J. et al.) (Wiley \& Sons, 2005)

11 Leibler, L. \& Sekimoto, K. On the sorption of gases and liquids in glassy-polymers. Macromolecules 26, 6937 (1993).

12 Parbhoo, B., Izrael, S., Salamanca, J. M. \& Keddie, J. L. Use of ellipsometry and gravimetry to develop calibration standards for measuring silicone coat weight and thickness with X-ray fluorescence spectroscopy. Surf. Interface Anal. 29, 341 (2000).

13 Worajittiphon, P., Jurewicz, I., King, A. A. K., Keddie, J. L. \& Dalton, A. B. Thermal actuation in thin polymer films through particle nano-squeezing by carbon nanotube belts. Adv. Mater. doi:10.1002/adma.201003145 (2010).

14 Kelley, F. N. \& Bueche, F. Viscosity and glass temperature relations for polymer-diluents systems. J. Polym. Sci. L 549 (1961).

15 Reiter, G. \& de Gennes, P. G. Spin-cast, thin glassy polymer films: highly metastable forms of matter. Eur. Phys. J. E 6, 25 (2001).

16 Hutchinson, J. M. Physical aging of polymers. Prog. Polym. Sci. 20, 703 (1995).

17 Simon, S. L., Bernazzini, P. \& McKenna, G. B. Effects of freeze-drying on the glass temperature of cyclic polystyrenes. Polymer 44, 8025 (2003).

18 Young, R. J. \& Lovell, P. A. Introduction to Polymers (CRC Press, London, 1991).

19 Elbs, H. \& Krausch, G. Ellipsometric determination of Flory-Huggins interaction parameter in solution. Polymer 45, 7935 (2004).

20 Wulf, M., Grundke, K., Kwok, D. Y. \& Neumann, A. W. Influence of different alkyl side chains on solid surfaces of polymethacrylate. J. Appl. Polym. Sci. 77, 2493 (2000).

21 Liang Cui, L., Ding, Y., Li, X., Wang, Z. \& Han, Y. Solvent and polymer concentration effects on the surface morphology evolution of immiscible polysterene/poly(methyl methacrylate) blends. Thin Solid Films 2038, 515 (2006).

22 Gourgon, C., Tortai, J. H., Lazzarino, F., Perret, C., Micouin, G., Joubert, O. \& Landis, S. Influence of residual solvent in polymers patterned by nanoimprint lithography. J. Vac. Sci. Tech. 22, 602 (2004). 